\title{
Worldwide Dyslipidemia Guidelines
}

\author{
Marcin Adam Bartlomiejczyk ${ }^{1}$ - Peter Penson ${ }^{2}$ - Maciej Banach ${ }^{1,3}$
}

Published online: 25 January 2019

(C) The Author(s) 2019

\begin{abstract}
Purpose of Review This review aims to identify common features of guidelines by comparing and summarizing similarities between five guidelines distributed by high-profile cardiovascular societies across the globe. We include guidelines from North America: ACC/AHA (2013 American College of Cardiology/American Heart Association Guideline on the Treatment of Blood Cholesterol to Reduce Atherosclerotic Cardiovascular Risk in Adults) and CCS (2016 Canadian Cardiovascular Society Guidelines for the Management of Dyslipidemia for the Prevention of Cardiovascular Disease in the Adult) and from Europe: PoLA (2016 Polish Lipid Association), ESC/EAS (2016 European Society for Cardiology/European Atherosclerosis Society Guidelines for the Management of Dyslipidemias), and NICE (National Institute for Health and Care Excellence) from the UK. We also include the 2016 Chinese guidelines for the management of dyslipidemia in adults in this comparison.

Recent Findings All of these guidelines employ a rigorous review of clinical evidence and emphasize the immense importance of statins in the primary and secondary prevention of atherosclerotic cardiovascular disease. Moreover, they place great emphasis on the dialog between the clinician and the patient regarding treatment and the risks associated with it.

Summary Despite the differences in statin intensities, safety concerns, use of risk estimators, or treatment of specific patient subgroups, there are more similarities than differences between the guidelines from both a clinical and practical point of view. Physicians ought to understand both similarities and differences in guideline recommendations to make the right decision regarding statin therapy for individual patients.
\end{abstract}

Keywords Dyslipidemia $\cdot$ Statin treatment $\cdot$ Clinical practice guidelines $\cdot$ Risk factors $\cdot$ Cardiovascular risk

\section{Introduction}

The standard definition of clinical practice guidelines (CPGs) is "systematically developed statements to assist practitioners and patient decisions about appropriate health care for specific circumstances" [1]. CPGs are based on evidence, literature data, and medical principles [2]. Understanding the limitations of accessible evidence and

This article is part of the Topical Collection on Lipids

Marcin Adam Bartlomiejczyk

marcin.bartlomiejczyk@umed.lodz.pl

1 Department of Hypertension, Chair of Nephrology and Hypertension, WAM University Hospital in Lodz, Medical University of Lodz, Zeromskiego 113, 90-549 Lodz, Poland

2 School of Pharmacy and Biomolecular Sciences, Liverpool John Moores University, Liverpool, UK

3 Polish Mother's Memorial Hospital Research Institute, Lodz, Poland an awareness of other available guidelines will support specialists to make personalized decisions with patients and improve the clinician-patient discussion [3, 4]. We need to create universal solutions for CPG on dyslipidemia to have guidelines that will simplify the decision-making process. In this paper, we consider five guidelines distributed by high-profile cardiovascular societies. From North America (American College of Cardiology/American Heart Association [ACC/AHA] [5••] and Canadian Cardiovascular Society [CCS] [6••]), Europe (European Society for Cardiology/European Atherosclerosis Society [ESC/EAS] [7••], Polish Lipid Association [PoLA] [8••], National Institute for Health and Care Excellence [NICE] $[9 \bullet \bullet])$, and Chinese guidelines $[10 \bullet \bullet$. In the later sections of the article, we will try to indicate the differences in statin intensities, treatment of specific patient subgroups, use of risk estimators, and consideration of safety concerns. This will enable clinicians to understand these differences and similarities, in order to make the most appropriate choices with respect to lipid-lowering therapy. 
Of note, the ACC/AHA plan to release updated lipid CPG in November 2018; at this time, it is uncertain whether the risk cut-points and treatment thresholds will differ from their prior 2013 guidelines, and whether they will re-incorporate a strategy of targeting to specific LDL-C reductions [11].

\section{Clinical Evidence in Guideline Recommendations and Risk Estimators}

Guidelines are based on information from various sources. In preparing their guidelines, the ACC/AHA took into account only randomized control trials (RCTs) and their systematic reviews and meta-analysis. Additionally, studies of poor quality were rejected [5••]. In contrast, the ESC/EAS and CCS did not specify restrictions on the types of studies, but they applied rigorous analysis to published data and recommendations $[6 \bullet \bullet, 7 \bullet \bullet]$. Meanwhile, PoLA considered results obtained from research conducted with a random selection of participants in the general population and studies conducted in patients using primary care $[8 \cdot \bullet]$. Chinese guidelines were created on the basis of clinical and epidemiological studies on the Chinese population, which were combined with international research and guidelines [10••]. NICE guidelines were based on systematic reviews and RCTs [9••]. Each guideline describes the strength for every recommendation using recommendation classes (e.g., I, IIa, IIb, and III) and the quality of the evidence (e.g., levels of evidence from A to C) that supports them $[6 \bullet \bullet, 7 \cdot \bullet, 8 \bullet \bullet, 9 \bullet \bullet, 10 \bullet \cdot]$.

All CPGs reviewed here recommend statins as a first-line drug therapy for primary prevention, although various estimators for a 10-year risk for atherosclerotic cardiovascular disease (ASCVD) events are advised. ACC/AHA Pooled Cohort Risk Equations are used by the ACC/AHA, while the Framingham Risk Score (FRS) is recommended by CCS $[5 \cdot \bullet, 6 \bullet, 12,13]$. In terms of predicted outcomes, the FRS is the most comprehensive, predicting 10 -year risk of coronary heart disease, peripheral artery disease, heart failure, or cerebrovascular events. The ACC/AHA Pooled Cohort Risk Equations are restrictive, predicting 10-year risk for a first hard ASCVD event, defined as coronary heart disease death, stroke, or nonfatal myocardial infarction (MI). In Europe, the Systematic Coronary Risk Evaluation (SCORE) estimator is recommended by the ESC/EAS, which has the strictest outcome by calculating the risk of only fatal events $[7 \bullet \bullet]$. The SCORE estimator is most specific, predicting 10-year risk of a first fatal atherosclerotic event, including stroke, MI, sudden cardiac death, or other occlusive arterial disease. PoLA uses updated risk assessment tables (created based on SCORE) tailored to the Polish population-Pol-SCORE 2015 [8••]. NICE recommends the use of the QRISK2 risk assessment tool for people under 85 years of age for primary prevention or people with T2DM (type 2 diabetes mellitus). They do not recommend using QRISK2 in people with T1DM (type 1 diabetes mellitus), chronic kidney disease (CKD), preexisting CVD, family burden of hypercholesterolemia, or other disorders of lipid metabolism. People over 40 years of age should be subjected to CVD risk assessment on an ongoing basis, based on data included in the primary care system relating to CVD risk factors [9*0]. Meanwhile, China based on the 2007 Blood Lipid Guidelines recommendation suggests using the risk of developing "ischemic cardiovascular disease" $[10 \bullet \bullet$. These differences in outcome measures are important when analyzing the differences in treatment thresholds between the guidelines. Risk estimators come from major studies and take into account predictors such as sex, age, high-density lipoprotein cholesterol (HDL-C), total cholesterol (TC), and systolic blood pressure. Nevertheless, diabetes, ethnicity, and treatment for hypertension are not universally taken into account in all CPGs, and therefore patient risk may be calculated differently depending on the estimators used.

\section{Common Aspects of Guidelines}

Comparing all the CPGs discussed here reveals that there are more similarities than differences between them from a clinical and a practical point of view. Examples of similarities between the discussed CPGs are as follows: (1) the use of statins as the first-line medications; (2) a preference for evidence from RCTs, when it is available; (3) the importance of discussion with patients in order to improve outcomes through shared decision-making [14] — especially when limited evidence from RCTs is available; (4) the suggestion that nonpharmacological measures are employed to enhance the treatment of dyslipidemia despite the fact that there is often relatively little RCT evidence to support such approaches (The PREDIMED trial, being a notable exception [15]); (5) management based on cardiovascular (CV) global risk considering interactions among all present in each patient.

With regard to individual CV risk estimators, all the guidelines are limited by the poor predictive power of calculators. Another common feature of the CPGs discussed here is the categorization of statins by their efficiency into low-, medium-, and high-intensity classes. "Intensity" is the best way to classify statins in guidelines, because different medications in comparable doses may show different intensities as defined as the level of lowdensity lipoprotein cholesterol (LDL-C) reduction. They are characterized by their ability to reduce pretreatment concentrations of LDL-C levels by $<30 \%, 30$ to 40 $50 \%$, and $>40-50 \%$, respectively. High-intensity statins should be used in patients with high LDL-C/non-highdensity lipoprotein cholesterol (non-HDL-C) levels, which need to be reduced because of a high individual $\mathrm{CV}$ risk. An exception to this approach is found in the 
Chinese guidelines in which statins were divided into two intensity groups: medium and high. They are characterized by the ability to lower LDL-C levels by $25-50 \%$ and $>50 \%$, respectively. Interestingly, there are also a lot of similarities in statin classification due to their effectiveness. However, there is not complete agreement between CPGs in this area. Table 1 illustrates the classification of statins by their ability to reduce plasma LDL-C levels. The CCS does not focus on statin intensity or dosing but rather on a targeted reduction in LDL-C level [6*0]. ACC/AHA and NICE recommend statin dose or intensity based on clinical profiles $[5 \bullet, 18]$. Discrepancies in statin classifying result from the use of various bibliographies during the creation of guidelines $[16,19,20]$. All guidelines include effectiveness as a criterion in the choice of statins to use. NICE conclude that atorvastatin should be used in primary and secondary prevention at the dose of 20 and $80 \mathrm{mg} /$ day, respectively.

\section{Primary Prevention}

Statin treatment is recommended by ESC/EAS if the patient has a 10-year ASCVD risk of 5 to $10 \%$ using SCORE risk estimator and LDL-C $\geq 2.5 \mathrm{mmol} / 1(100 \mathrm{mg} / \mathrm{dl})$ [7••]. The CCS suggest a threshold of $\geq 20 \%$ 10-year ASCVD risk (the higher one) because of using FRS estimator [6••]. Using the 2013 ACC/AHA Pooled Cohort Risk Equations, various CPGs in the USA have adopted differing thresholds of $\geq$ $7.5 \%$ (ACC/AHA), $\geq 10 \%$ (USPSTF), and $\geq 12 \%$ (VA/DoD) 10 -year risk of ASCVD respectively [6••, 12, 13]. Whereas in Chinese guidelines, it is $<5 \%, 5-9 \%$, and $\geq 9 \% 10$-year risk of ASCVD for low, medium, and high risk, respectively [10••]. According to Pol-SCORE, PoLA have adopted a threshold of $<1 \%, 1-5 \%, \geq 5-<10 \%$, and $\geq 10 \%$ for low, medium, high, and very high risk, respectively [8••].

Simultaneously, all CPGs recommend treatment for patients with LDL-C level $\geq 4.9 \mathrm{mmol} / \mathrm{l}(190 \mathrm{mg} / \mathrm{dl})$. The

Table 1 Classification of statins due to their ability to reduce LDL-C (daily dose)

\begin{tabular}{|c|c|c|c|}
\hline & Low intensity & Medium intensity & High intensity \\
\hline \multirow[t]{2}{*}{ ACC/AHA [5••] } & $<30 \%$ LDL-C reduction & $30-49 \%$, LDL-C reduction & $\geq 50 \%$, LDL-C reduction \\
\hline & $\begin{array}{l}\text { Fluv.: } 20-40 \mathrm{mg} \text {, Lova.: } 20 \mathrm{mg} \text {, } \\
\text { Prav.: } 10-20 \mathrm{mg} \text {, Simv.: } 10 \mathrm{mg} \text {, } \\
\text { Pita.: } 1 \mathrm{mg}\end{array}$ & $\begin{array}{l}\text { Fluv.: } 40 \mathrm{mg} \text {, Fluv.: ex } 80 \mathrm{mg} \text {, } \\
\text { Prav.: } 40-80 \mathrm{mg} \text {, Lova.: } 40 \mathrm{mg} \text {, } \\
\text { Simv.: } 20-40 \mathrm{mg} \text {, Ator:. } 10-20 \mathrm{mg} \text {, } \\
\text { Rosu.: } 5-10 \mathrm{mg} \text {, Pita.: } 2-4 \mathrm{mg}\end{array}$ & Ator:: $80 \mathrm{mg}$, Rosu.: $20-40 \mathrm{mg}$ \\
\hline \multirow[t]{2}{*}{ ESC/EAS $[16,17]$} & 20-30\%, LDL-C reduction & $31-40 \%$, LDL-C reduction & $>40 \%$, LDL-C reduction \\
\hline & $\begin{array}{l}\text { Fluv.: } 40 \mathrm{mg} \text {, Prav.: } 20-40 \mathrm{mg} \\
\text { Lova.: } 10-20 \mathrm{mg} \text {, } \\
\text { Simv.: } 10 \mathrm{mg}\end{array}$ & $\begin{array}{l}\text { Fluv.: } 80 \mathrm{mg} \text {, Lova.: } 40-80 \mathrm{mg} \text {, } \\
\text { Simv.: } 20 \mathrm{mg} \text {, Ator.: } 10 \mathrm{mg} \text {, } \\
\text { Pita.: } 1 \mathrm{mg}\end{array}$ & $\begin{array}{l}\text { Ator:: } 40 \mathrm{mg} \text {, Rosu.: } 40 \mathrm{mg} \text {, } \\
\text { Pita.: } 2-4 \mathrm{mg}\end{array}$ \\
\hline \multirow[t]{2}{*}{ NICE $[9 \bullet \bullet]$} & 20-30\%, LDL-C reduction & $31-40 \%$, LDL-C reduction & $>40 \%$, LDL-C reduction \\
\hline & $\begin{array}{l}\text { Ator.: } 20 \mathrm{mg} \text {, Fluv.: } 20-40 \mathrm{mg} \text {, } \\
\text { Prav.: } 10-40 \mathrm{mg} \text {, Simv.: } 10 \mathrm{mg}\end{array}$ & $\begin{array}{l}\text { Fluv.: } 80 \mathrm{mg} \text {, Simv.: } 20-40 \mathrm{mg} \text {, } \\
\text { Ator.: } 10 \mathrm{mg} \text {, Rosu.: } 5 \mathrm{mg}\end{array}$ & $\begin{array}{l}\text { Simv.: } 80 \mathrm{mg} \text {, Ator.: } 20-80 \mathrm{mg} \text {, } \\
\text { Rosu.: } 10-40 \mathrm{mg}\end{array}$ \\
\hline \multirow[t]{2}{*}{ PoLA $[8 \bullet \bullet]$} & $<50 \%$ LDL-C reduction & Min. 50\%, LDL-C reduction & $50-60 \%$, LDL-C reduction \\
\hline & $\begin{array}{l}\text { Simv.: } 20-40 \mathrm{mg} \text {, Ator.: } 10-20 \mathrm{mg} \text {, } \\
\text { Rosu.: } 5-10 \mathrm{mg}\end{array}$ & $\begin{array}{l}\text { Ator:: 20-40 mg, Rosu.: 10-20 mg, } \\
\text { Simv.: 20-40 mg + Ezet.: } 10 \mathrm{mg}, \\
\text { Ator.: } 10-20 \mathrm{mg} \text { + Ezet.: } 10 \mathrm{mg} \\
\text { Rosu.: } 5-10 \mathrm{mg} \text { + Ezet.: } 10 \mathrm{mg} \text {, } \\
\text { Ator.: } 40 \mathrm{mg} \text { + Ezet.: } 10 \mathrm{mg} \text {, } \\
\text { Rosu.: } 20 \mathrm{mg} \text { + Ezet.: } 10 \mathrm{mg}\end{array}$ & $\begin{array}{l}\text { Ator:: 40-80 mg, Rosu.: } 20-40 \mathrm{mg} \text {, } \\
\text { Simv.: } 40 \mathrm{mg} \text { + Ezet.:10 mg, } \\
\text { Ator:: } 20 \mathrm{mg} \text { + Ezet.: } 10 \mathrm{mg}, \\
\text { Rosu.: } 10 \mathrm{mg} \text { + Ezet.: } 10 \mathrm{mg}, \\
\text { Ator.: } 40-80 \mathrm{mg} \text { + Ezet.: } 10 \mathrm{mg} * * \text {, } \\
\text { Rosu.: } 20-40 \mathrm{mg} \text { + Ezet.: } 10 \mathrm{mg} * *\end{array}$ \\
\hline \multirow[t]{2}{*}{$\operatorname{CCS}[6 \bullet \bullet]$} & \multicolumn{3}{|c|}{ Does not focus on statin intensity or dosing but rather on a targeted reduction in LDL-C level } \\
\hline & \multicolumn{3}{|c|}{$\begin{array}{l}\text { Ator.: } 10-80 \mathrm{mg} \text {, Fluv.: } 20-80 \mathrm{mg} \text {, } \\
\text { Lova.: } 20-80 \mathrm{mg} \text {, Prav.: } 10-40 \mathrm{mg} \\
\text { Rosu.: } 5-40 \mathrm{mg} \text {, Simv.: } 10-80 \mathrm{mg}\end{array}$} \\
\hline \multirow[t]{2}{*}{ Chinese $[10 \bullet \bullet]$} & \multirow[t]{2}{*}{ No recommendations } & $25-50 \%$, LDL-C reduction & $\geq 50 \%$, LDL-C reduction \\
\hline & & $\begin{array}{l}\text { Ator.: } 10-20 \mathrm{mg} \text {, Rosu.: } 5-10 \mathrm{mg} \text {, } \\
\text { Fluv.: } 80 \mathrm{mg} \text {, Lova.: } 40 \mathrm{mg} \text {, } \\
\text { Pita.: } 2-4 \mathrm{mg} \text {, Prav.: } 40 \mathrm{mg} \text {, } \\
\text { Simv.: } 20-40 \mathrm{mg} \text {, Xuez.: } 1.2 \mathrm{~g}\end{array}$ & Ator:: $40-80$ mg*, Rosu.: 20 mg \\
\hline
\end{tabular}

ACC/AHA, 2013 American College of Cardiology/American Heart Association Guideline on the Treatment of Blood Cholesterol to Reduce Atherosclerotic Cardiovascular Risk in Adults; CCS, 2016 Canadian Cardiovascular Society Guidelines for the Management of Dyslipidemia for the Prevention of Cardiovascular Disease in the Adult; PoLA, 2016 Polish Lipid Association; ESC/EAS, 2016 European Society for Cardiology/European Atherosclerosis Society Guidelines for the Management of Dyslipidemias; NICE, National Institute for Health and Care Excellence; Ator., Atorvastatin; Ezet., Ezetimibe; Fluv., Fluvastatin; Lova., Lovastatin; Pita., Pitavastatin; Prav., Pravastatin; Rosu., Rosuvastatin; Simv., Simvastatin; Xuez., Xuezhikang; ex, extended release. * in the case of baseline LDL-C $>190 \mathrm{mg} / \mathrm{dl}(4.9 \mathrm{mmol} / \mathrm{l})$ very intensive hypolipidemic therapy (LDL-C reduction $>60 \%), * *$ very intensive hypolipidemic therapy (LDL-C reduction $>60 \%$ ) 
guidelines emphasize the importance of lifestyle (e.g., avoiding smoking, reducing excessive weight, heart-healthy diets, and physical exercise) before and in combination with pharmacotherapy to reduce the risk of ASCVD. Moreover, in all guidelines, it is clearly stated that statins should be considered to be first-line medicines during pharmacological treatment. In primary prevention, all CPGs put great emphasis on joint decision-making and discussion between physicians and patients. Based upon the 10-year risk thresholds, the CPGs recommend different dosages and intensities of statins for primary prevention. The ACC/AHA guidelines indicate the use of high- or moderate-intensity statin therapy (for patients with $\geq 7.5 \%$ 10-year ASCVD risk). The ESC/EAS and CCS regulate the selection and dosing of statins by treatment goals [5••, $6 \bullet \bullet, 7 \bullet \bullet, 12,13]$. Interestingly, based on HPS2-THRIVE results, Chinese people may achieve lower LDL-C levels compared to Europeans when using the same drugs and dosing regimens [21]. Furthermore, higher doses do not affect the effectiveness of LDL-C lowering as demonstrated in the CHILLAS and DYSISCHINA studies [22, 23].

\section{Secondary Prevention}

In secondary prevention, the ACC/AHA suggest variable intensities or doses of statins in patients with ASCVD. Patients over 75 years old without contraindications should receive high-intensity statins whereas others should obtain moderate-intensity statins [5••]. Meanwhile, ESC/EAS and CCS select the dose of statin based on therapeutic goals [6••, $7 \cdot \bullet]$. All of the guidelines agree with each other that the main adverse effects (hepatitis, diabetes, and myopathy) have a marginally increased frequency of occurrence in statintreated patients [24], but that benefits outweigh potential harms. Results from systematic reviews have shown that the true incidence of adverse effects is unknown and can be hard to measure. However, in general, the long-term safety profile of lipid-lowering therapy is good $[8 \cdot \bullet, 9 \bullet \bullet, 10 \bullet \bullet, 25,26]$. Furthermore, all guidelines agree with each other that is a need of baseline and periodic analytical monitoring for early detection of adverse effects. On account of the low incidence of adverse effects, guidelines recommend monitoring only patients at risk or with clinical manifestation [27].

Future guidelines, such as the planned release of the 2018 ACC/AHA lipid guidelines, are likely to incorporate some recommendations for add-on therapies, including ezetimibe and/or PCSK9-inhibitors, for secondary prevention in patients who have not achieved the targeted LDL-C reduction on maximally tolerated statin doses.

\section{Monitoring Recommendations and Safety Concerns}

Interestingly, the ESC/EAS guidelines state that a very important factor in counteracting liver and muscle damage is the ability to identify people at high risk. Risk factors include the following: small body size, advanced age, hepatic and renal dysfunction, female sex, multisystem disease, hypothyroidism, perioperative periods, and alcohol abuse [27, 28]. Similar risk factors are considered by the ACC/AHA guideline such as impaired hepatic or renal function, hemorrhagic stroke, Asian ancestry, age over 75 years, previous muscle disorders, or statin intolerance or simultaneous use of drugs affecting statin metabolism [29]. All discussed CPGs recommend monitoring of transaminases in each patient before starting treatment with statins. Moreover, monitoring of the biomarker creatine kinase (CK) is recommended by all of the discussed guidelines. ESC/EAS, PoLA, and Chinese guidelines recommend monitoring $\mathrm{CK}$ in all patients [7••, $8 \bullet \bullet, 10 \bullet \bullet, 12]$. However, ACC/AHA [29] suggest baseline monitoring only in patients with muscular symptoms, and those at risk for myopathy (family or personal burden of muscle disease or statin intolerance or drug therapy which can increase the risk of myopathy). NICE recommends checking CK before starting statin treatment only in some circumstances and after including assessment such as smoking status, blood pressure, alcohol consumption, body mass index (BMI), TC, non-HDL-C, HDL-C and triglycerides, HbAlc, renal function and eGFR (estimated glomerular filtration rate), transaminase level (alanine aminotransferase or aspartate aminotransferase), and thyroid stimulating hormone [18]. With respect to ongoing monitoring of therapy, the ACA/AHA guidelines [29] recommend measurement of CK and transaminases, only if symptoms occur, e.g., jaundice (suggesting hepatotoxicity) or symptoms suggesting myotoxicity (tenderness, cramping, pain, stiffness, general fatigue, or weakness). In the ESC/EAS, PoLA, and Chinese $[8 \bullet \bullet, 27,28]$ recommendations, it is stated that CK levels should be measured only if muscle symptoms occur after the initiation of statins. Unlike ACC/AHA guidelines [29], transaminases should be checked 8 weeks after starting statin therapy and once a year if its values are three times lower than normal value. NICE guidelines suggest that transaminases are always measured at base-

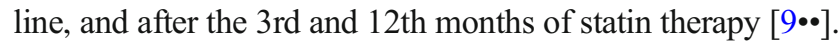
Liver and muscle damage, requiring cessation of therapy, are indicated by elevations of transaminase and CK to three and five times, respectively, the upper limit of normal.

The CPGs differ in their reasons for recommending the monitoring of lipid profiles. The ESC/EAS, Chinese, and PoLA $[8 \bullet \bullet, 10 \bullet, 18,27,28]$ require plasma lipids to be measured, because they are the treatment targets. Lipid monitoring can also be useful in aiding and measuring compliance to lipid-lowering therapy. Coodley et al. have commented "A separate issue is the impact of regular lipid monitoring in promoting patient adherence to lifestyle changes or drug regimens that impact positively on their health, as found in a range of studies. It is unclear if only the process of monitoring is critical in achieving this or a combination of education, 
regular contact, and adherence assessment" [17]. The 2013 ACC/AHA guidelines recommend monitoring to ensure that LDL-C level has decreased and to assess the therapeutic adherence of the treated person [29]. NICE also considers pharmacological adherence as a cornerstone of the management of $\mathrm{CV}$ risk but has not outlined any specific and effective specific strategies to increase the adherence to statin treatment [9・•]. For ESC/EAS and PoLA guidelines, the elementary lipid profile includes TC (total cholesterol), TG (triglyceride), HDL-C, LDL-C, non-HDL-C, and TC/HDL-C ratio [8・•, 27, 28]. ACC/AHA and NICE suggest TC, TG, HDL-C, LDL-C, and non-HDL-C $[9 \bullet \cdot, 29]$. NICE is unique in not recommending a fasting blood sample [9*0]. Chinese guidelines not only recommend the measurement of TC, LDL-C, TG, and HDL-C but also include other blood lipids such as lipoprotein(a) and apolipoprotein (apo) A1 or B [10••]. All guidelines advise the periodic monitoring of analytical profiles after starting statin therapy. ACC/AHA guidelines recommend monitoring after 4-12 weeks of statin therapy and then every 3-12 months [29]. ESC/EAS recommend tests after 1-12 weeks of statin therapy, 3-4 weeks after changing medications, and once a year after reaching the therapeutic goal $[27,28]$. NICE guidelines advise the determination of lipid profiles 3 months after starting lipid-lowering therapy and then once a year thereafter $[9 \cdot \bullet]$. In the case of acute coronary events, testing should be performed 4 weeks after initiation of lipid-lowering therapy. Interestingly, a metaanalysis performed by Perera et al. undermined the CPGs by showing that testing once a year is the most predictive as well as effective time period for monitoring lipids [30 $]$.

\section{Special Groups of Patients}

Special groups of patients include the elderly (age over 75 years old), who owing to the presence of various comorbidities and altered pharmacokinetic of drugs are at high risk of drug interactions. All of the CPGs discussed in this article highlight uncertainty around the use of statins in the elderly. The ACC/AHA does not contraindicate the use of statins if they are well tolerated, but at the same time does not recommend that they be initiated in primary prevention in this group. They also suggest that statins of moderate intensity should be used in secondary prevention [5••]. The ESC/EAS, PoLA, and Chinese guidelines suggest starting statin therapy for primary prevention if ASCVD risk is high. All guidelines recommend starting treatment with low-dose statins and then carefully increasing the dose until the target level of LDL-C (or target intensity of treatment) is reached $[7 \bullet \bullet, 8 \bullet \bullet, 9 \bullet \bullet, 10 \bullet \cdot]$. Furthermore, all CPGs highlight the importance of preventing cardiovascular diseases in the elderly by the promotion of a healthy lifestyle. Interestingly, Chinese guidelines recommended monitoring the kidney and liver function as well as CK in the elderly [10•*]. Another special group of patients is people with kidney disease. The ACC/AHA does not make specific recommendations relating to the use of statins in patients with end-stage renal disease on maintenance hemodialysis [5••]. The CCS recommend that patients on dialysis should not initiate new statin therapy, but that they should continue existing statin therapy $\left[6^{\bullet \bullet}\right]$. On the other hand, according to PoLA, ESC/EAS, and Chinese guidelines, the decision should depend on the assessment of total cardiovascular risk based on the age of the patient and the degree of renal failure and/or estimated glomerular filtration rate (eGFR) [8••]. The KIDIGO guidelines (Kidney Disease: Improving Global Outcomes) determine the doses of optimal statins for individual stages of chronic renal failure, and not dependent on the LDL-C values. In this group of patients, medications excreted via hepatic metabolism (atorvastatin, fluvastatin, pitavastatin, ezetimibe) are preferred. Statins metabolized by CYP3A4 may lead to side effects due to multiple drug interactions and are not therefore recommended [8••].

Another special group of patients is people with human immunodeficiency virus (HIV) and recipients of solid organ transplants. ESC/EAS and PoLA suggest caution with drug/drug interactions and suggest starting statin therapy with low doses and then gradually increasing. Both organizations recommend caution and clinical judgment before statin treatment is initiated in patients with inflammatory and rheumatologic diseases. Furthermore, they cite research showing that in palliative patients, discontinuation of statin therapy was not associated with deterioration in the assessment of cardiovascular parameters, including mortality; but significantly improved the quality of life of these patients [31]. Clinicians should always consider an individual approach to the patient, remembering that discontinuation of statin therapy might be associated with an increased risk of cardiovascular events [32, 33•]. Particular attention should be paid to the interaction of statins with protease inhibitors in patients with HIV due to metabolism by CYP3A4, leading to an increased risk of myopathy and rhabdomyolysis [7••]. While TC and LDL-C concentrations are often reduced in these patient groups, treatment may negatively affect the lipid profile. Highly active antiretroviral therapy (HAART), including primarily protease inhibitors, has a negative effect on the lipid profile, particularly on the development of atherogenic dyslipidemia [31]. If such lipid disorders are found, lipid-lowering medication may be considered as part of HAART, and pravastatin may be considered as recommended for HIV patients due to minimal metabolism through the cytochrome P450 isoenzyme system. In addition to pravastatin, pitavastatin, atorvastatin, fluvastatin, and rosuvastatin may also be considered. It is also worth noting that the cardiovascular risk of a patient with HIV is higher than that of a patient without HIV (>60\%), and antiretroviral drugs, in particular protease inhibitors, increase this risk up to two-fold $[8 \bullet \bullet$. ESC/EAS guidelines do not make any recommendations for people with psychiatric disorders as a barrier 
to drug compliance, owing to the lack of unambiguous data confirming the effectiveness of statin therapy in this population $[5 \cdot \bullet, 7 \bullet \bullet$.

\section{Issues to Be Improved in CPG Regarding Lipids}

Aside from the minor differences between guidelines, we can identify many common features in the various GCP recommendations. These can be implemented as a starting point for creating a common strategy. Consideration should be given to (1) the need of treating global CV risk instead of individual risk factors; (2) the need to continue to gather clinical evidence to support the use of non-statin lipid-lowering drugs, (3) the need to gain confirmation from RCTs on nonpharmacological treatments for dyslipidemia.

Only the ESC/EAS in its guidelines mentions that "systematic comparison of current international guidelines to define areas of agreement and the reasons for discrepancies." [27, 28]. On the other hand, only NICE highlights the need for more data on effectiveness $[9 \bullet \cdot$. There is a complete agreement on the need to obtain more data about the benefits of therapy, especially for older people, women, and diabetic patients. The main goal in the treatment of dyslipidemias is to avoid CV events. The use of non-HDL-C or LDL-C as targets or predictors of risk needs to be promoted without losing sight of the importance of managing global CV risk. Lipid parameters should be monitored, because clinicians make treatment decisions based on the numerical values of the results that distinguish healthy patients from ill patients. A reduction in global incidence of $\mathrm{CV}$ events could result from the effective implementation of evidence-based guidelines [34].

\section{Conclusions}

To sum up, there are more similarities than differences between the CPGs compared in this review, from both clinical and practical point of view. All of the CPGs recommend statins for primary and secondary prevention. Furthermore, they all recommend shared decision-making between the patient and the clinician. Taking all the similarities together, the recommended practical approach for the regular medical practice should be based on (1) early detection of people with an increased CV risk (promoting the use of approved local riskprediction tools); (2) strengthening the mainstream importance of nonpharmacological treatment for the reduction of CV Risk; (3) the need for global CV risk estimation and periodic monitoring of therapeutic response with analytical parameters (non-high-density lipoprotein cholesterol or lowdensity lipoprotein).

However, there are also differences between the guidelines, for example, in treatment of patients with comorbidities, statin intensity or in safety concerns. Moreover, using different risk estimators requires an understanding of compounding comorbidities and their impact on the occurrence of ASCVD. Globalization, technology development, and solutions (e.g., big data) will be useful in acquiring high-quality evidence on the basis of which it will be possible to develop better and better GCP. We hope that in the near future, we will succeed in developing consistent international guidelines.

Funding This review was written independently; no company or institution supported it financially. No professional writer was involved in the preparation of this meta-analysis.

\section{Compliance with Ethical Standards}

Conflict of Interest Maciej Banach has served on the speakers bureau of Abbott/Mylan, Abbott Vascular, Actavis, Akcea, Amgen, Biofarm, KRKA, MSD, Sanofi-Aventis, Servier, and Valeant and has served as a consultant to Abbott Vascular, Akcea, Amgen, Daichii Sankyo, Esperion, Lilly, MSD, Resverlogix, Sanofi-Aventis; grants from Sanofi and Valeant; Peter Penson owns four shares in AstraZeneca PLC and receives Speaker's fees from Amgen Inc.; Marcin A. Bartlomiejczyk has no conflicts of interest to disclosure.

Human and Animal Rights and Informed Consent This article does not contain any studies with human or animal subjects performed by any of the authors.

Open Access This article is distributed under the terms of the Creative Commons Attribution 4.0 International License (http:// creativecommons.org/licenses/by/4.0/), which permits unrestricted use, distribution, and reproduction in any medium, provided you give appropriate credit to the original author(s) and the source, provide a link to the Creative Commons license, and indicate if changes were made.

Publisher's Note Springer Nature remains neutral with regard to jurisdictional claims in published maps and institutional affiliations.

\section{References}

Papers of particular interest, published recently, have been highlighted as:

- Of importance

•. Of major importance

1. Field MJ, Lohr KN. Guidelines for clinical practice: from development to use. Washington, D.C: Institute of Medicine, National Academy Press; 1992.

2. Rosenberg W, Donald A. Evidence based medicine: an approach to clinical problem-solving. BMJ. 1995;310(6987):1122-6.

3. Martin SS, Sperling LS, Blaha MJ, Wilson PWF, Gluckman TJ, Blumenthal RS, et al. Clinician-patient risk discussion for atherosclerotic cardiovascular disease prevention: importance to implementation of the 2013 ACC/AHA guidelines. J Am Coll Cardiol. 2015;65(13):1361-8.

4. Morris PB, McLain K. What the guidelines do not say: statin nonbenefit groups. Curr Atheroscler Rep. 2015;17(1):468.

5.• Stone NJ, Robinson JG, Lichtenstein AH, et al. 2013 ACC/AHA guideline on the treatment of blood cholesterol to reduce atherosclerotic cardiovascular risk in adults: a report of the American 
College of Cardiology/American Heart Association Task Force on Practice Guidelines. J Am Coll Cardiol. 2014;63(25 Pt B):2889934 Currently available guidelines for managing dyslipidemia in the USA.

6.• Anderson TJ, Gregoire J, Pearson GJ, et al. 2016 Canadian Cardiovascular Society guidelines for the management of dyslipidemia for the prevention of cardiovascular disease in the adult. Canadian J Cardiol. 2016;32:1263-82 Currently available guidelines for managing dyslipidemia in Canada.

7.• Catapano AL, Graham I, De Backer G, et al. 2016 ESC/EAS guidelines for the management of dyslipidaemias. Eur Heart J. 2016;37: 2999-3058 Recent guidelines on dyslipidemia management in Europe.

8.• Banach M, Jankowski P, Jóźwiak J, et al. PoLA/CFPiP/PCS guidelines for the management of dyslipidaemias for family physicians 2016. Arch Med Sci. 2017;13(1):1-45 Recent guidelines on dyslipidemia management in Poland.

9.• Cardiovascular disease: risk assessment and reduction, including lipid modification. Clinical guideline. Published: 18 July 2014. nice.org.uk/guidance/cg181. Recent guidelines on dyslipidemia management in the United Kingdom of Great Britain and Northern Ireland.

10.• Joint committee for guideline revision, Zhao S-P, Lu G-P, Zhao D, Li J-J. 2016 Chinese guidelines for the management of dyslipidemia in adults. J Geriatr Cardiol. 2018;15:1-29 Recent guidelines on managing dyslipidemia in China available in English.

11. Baer J., AACE and EAS lipid guidelines. American College of Cardiology. 2017. https://www.acc.org/latest-in-cardiology/ articles/2017/08/11/08/35/aace-and-eas-lipid-guidelines. Accessed 05 Nov 2018.

12. Bibbins-Domingo K, Grossman DC, Curry SJ, et al. US Preventive Services Task Force. Statin use for the primary prevention of cardiovascular disease in adults: US Preventive Services Task Force Recommendation Statement. JAMA. 2016;316:1997-2007.

13. Downs JR, O'Malley PG. Management of dyslipidemia for cardiovascular disease risk reduction: synopsis of the 2014 U.S. Department of Veterans Affairs and U.S. Department of Defense Clinical Practice Guideline. Ann Intern Med. 2015;163(4):291-7.

14. Barratt A. Evidence based medicine and shared decision making: the challenge of getting both evidence and preferences into health care. Patient Educ Couns. 2008;73(3):407-12.

15. Martínez-González MA, Salas-Salvadó J, Estruch R, Corella D, Fitó M, Ros E, et al. Benefits of the mediterranean diet: insights from the PREDIMED study. Prog Cardiovasc Dis. 2015;58(1):50-60.

16. Weng TC, Yang YH, Lin SJ, Tai SH. A systematic review and metaanalysis on the therapeutic equivalence of statins. J Clin Pharm Ther. 2010;35(2):139-51.

17. Coodley GO, Jorgensen M, Kirschenbaum J, Sparks C, Zeigler L, Albertson BD. Lowering LDL cholesterol in adults: a prospective, community-based practice initiative. Am J Med. 2008;121(7):604-10.

18. Rabar S, Harker M, O'Flynn N, Wierzbicki AS, Guideline Development Group. Lipid modification and cardiovascular risk assessment for the primary and secondary prevention of cardiovascular disease: summary of updated NICE guidance. BMJ. 2014;349:g4356.

19. Cholesterol Treatment Trialists' (CTT) Collaboration, Baigent C, Blackwell L, Emberson J, et al. Efficacy and safety of more intensive lowering of LDL cholesterol: a meta-analysis of data from 170, 000 participants in 26 randomised trials. Lancet. 2010;376(9753): 1670-81.

20. Law MR, Wald NJ, Rudnicka AR. Quantifying effect of statins on low density lipoprotein cholesterol, ischaemic heart disease, and stroke: systematic review and meta-analysis. BMJ. 2003;326(7404):1423-0.

21. HPS2-THRIVE Collaborative Group. HPS2-THRIVE randomized placebo-controlled trial in 25673 high-risk patients of ER niacin/ laropiprant: trial design, pre-specified muscle and liver outcomes, and reasons for stopping study treatment. Eur Heart J. 2013;34:1279-91.

22. Zhao SP, Wang Y, Mu Y, Yu B, Ye P, Yan X, et al. Prevalence of dyslipidaemia in patients treated with lipid-lowering agents in China: results of the DYSlipidemia International Study (DYSIS). Atherosclerosis. 2014;235:463-9.

23. Zhao SP, Peng DQ, Yu BL, et al. The effect of moderatedose versus double-dose statins on patients with acute coronary syndrome in China: results of the CHILLAS trial. Atherosclerosis. 2014;233:707-12.

24. Marshall TM. New insights into the statin-cholesterol controversy. J Am Phys Surg. 2014;19(2):42-6.

25. McKenney JM, Davidson MH, Jacobson TA, Guyton JR. National Lipid Association Statin Safety Assessment Task Force. Final conclusions and recommendations of the National Lipid Association Statin Safety Assessment Task Force. Am J Cardiol. 2006;97(8A): 89C-95C.

26. Sattar N, Preiss D, Murray HM, Welsh P, Buckley BM, de Craen AJM, et al. Statins and risk of incident diabetes: a collaborative meta-analysis of randomized statin trials. Lancet. 2010;375(9716): $735-42$.

27. Reiner Z, Catapano AL, De Backer G, et al. European Association for Cardiovascular Prevention \& Rehabilitation, ESC Committee for Practice Guidelines (CPG) 2008-2010 and 2010-2012 Committees. ESC/EAS guidelines for the management of dyslipidaemias: the task force for the management of dyslipidaemias of the European Society of Cardiology (ESC) and the European Atherosclerosis Society (EAS). Eur Heart J. 2011;32(14):1769-818.

28. Perk J, De Backer G, Gohlke H, et al; European Association for Cardiovascular Prevention \& Rehabilitation (EACPR); ESC Committee for Practice Guidelines (CPG). European guidelines on cardiovascular disease prevention in clinical practice (version 2012). The Fifth Joint Task Force of the European Society of Cardiology and other societies on cardiovascular disease prevention in clinical practice. Eur Heart J 2012; 33(13): 1635-1701.

29. Stone NJ, Robinson JG, Lichtenstein AH, Bairey Merz CN, Blum $\mathrm{CB}$, Eckel RH, et al. American College of Cardiology/American Heart Association Task Force on Practice Guidelines. 2013 ACC/ AHA guideline on the treatment of blood cholesterol to reduce atherosclerotic cardiovascular risk in adults: a report of the American College of Cardiology/American Heart Association Task Force on Practice Guidelines. Circulation. 2014;129(25 Suppl 2):S1-S45.

30. Perera R, McFadden E, McLellan J, et al. Optimal strategies for monitoring lipid levels in patients at risk or with cardiovascular disease. A systematic review with statistical and cost-effectiveness modelling. Health Technol Assess. 2015;19(100):1-402 Systematic review about determining clinical value and costeffectiveness of different monitoring intervals and different lipid measures for primary and secondary prevention of CVD.

31. Melzi S, Carenzi L, Cossu MV. i wsp.: Lipid metabolism and cardiovascular risk in HIV-1 infection and HAART: present and future problems. Cholesterol. 2010;2010:271-504.

32. Banach M, Serban MC. Discussion around statin discontinuation in older adults and patients with wasting diseases. J Cachexia Sarcopenia Muscle. 2016;7(4):396-69.

33. Banach M, Rizzo M, Toth P. i wsp.: Statin intolerance - an attempt at a unified definition. Position paper from an International Lipid Expert Panel. Arch Med Sci. 2015;11(1):1-23 Position paper from an International Lipid Expert Panel about a unified definition of statin intolerance, and to complement the recent EAS statement on SAMS, where the pathophysiology, diagnosis, and the management were comprehensively presented.

34. Mendis S, Puska P, Norrving B, et al. Global atlas on cardiovascular disease prevention and control. Geneva: World Health Organization in collaboration with the World Heart Federation and the World Stroke Organization; 2011. 